in Russia (the results of comprehensive medical and statistical research) // Health of the Russian Federation. - 2012. - № 5. P. 21-26.

3. Report on the state of health in Europe 2002 // Regional Office WHO for Europe. - Copenhagen, 2002. - 70 p.

4. Namazova L.S., Modestov A.A., Torshkhoyeva R.M., Dybunova E.L. The prevalence of allergic diseases in children in the federal districts of the Russian Federation // Directory of Pediatrician. - 2007. - August. - P. 13-17.

5. On the sanitary-epidemiological situation in the Russian Federation in 2010: State report. - M.: Federal Center of Hygiene and Epidemiology RosPotrebNadzor, 2011. - 431 p.

6 . Key indicators of environmental protection. Statistical Bulletin. - M., 2011. - 116 p.

7. Basics towards Health for all in the WHO European Region. - Update 2005 - European Series towards health for all, № $7 .-98 \mathrm{p}$.

\section{Сведения об авторах}

Балаболкин Иван Иванович - gоктор медицинских наук, профессор, членкорр. РАМН, главный научный сотрудник отяеления пульмонологии и аляергологии, ФГБНУ «Научный центр зgоровья gетей».
Agрес: 119991, г. Москва, Аомоносовский проспект, g. 2; тел. 8(499) 1340607; e-mail: balabolkin@nczd.ru.

Терлецкая Римма Николаевна - gоктор меgицинских наук, профессор, главный научный сотруяник отgела социальной пеgиатрии, ФГБНУ «Научный центр зяоровья gетей».

Agрес: 119991, г. Москва, Аомоносовский проспект, g. 2; тел. 8(495) 9671571; e-mail: rterletskaya@mail.ru.

Моgестов Арсений Арсеньевич - gоктор меgицинских наук, профессор, завеяующий лаборатории организации медицинской помощи gетскому населению, ФГБНУ «Научный центр зуоровья gетей».

Agрес: 119991, г. Москва, Аомоносовский проспект, g. 2; тел. 8(495) 9671571; e-mail:modestov@yandex.ru.

\section{Authors}

Balabolkin Ivan Ivanovich - MD, PhD, Dr Med Sci, Project Leader. Pulmonology and Allergology Dept. Federal public budgetary scientific institution «Scientific Centre of Children Health".

Address: 2, Lomonosovskiy prospect, Moscow, 119991, Russia; Phone: (499) 1340607; e-mail: balabolkin@nczd.ru.

Terletskaya Rimma Nikolayevna - MD, PhD, Dr Med Sci, Project Leader, Dept. of Social Pediatrics. Federal public budgetary scientific institution «Scientific Centre of Children Health".

Address: 2, Lomonosovskiy prospect, Moscow, 119991, Russia; Phone: (495) 9671571; e-mail: rterletskaya@mail.ru.

Modestov Arseniy Arsenievich - MD, PhD, Dr Med Sci, Project Leader, Dept. of Social Pediatrics. Federal public budgetary scientific institution «Scientific Centre of Children Health».

Address: 2, Lomonosovskiy prospect, Moscow, 119991, Russia; Phone: (495) 9671571; e-mail:modestov@yandex.ru.

(c) ЯКОВАЕВ Я. Я., МАНЕРОВ Ф. К.

\title{
УАK 618.73-037
}

\section{ОЦЕНКА ФАКТОРОВ РИСКА ААКТОСТАЗА И ААКТАЦИОННОГО МАСТИТА}

Я. Я. Яковлев, Ф. К. Манеров

ГБОУ АПО Новокузнецкий государственный институт усовершенствования врачей Министерства здравоохранения РФ, ректор - А. м. н., проф. А. В. Колбаско; кафедра педиатрии и неонатологии, зав. - А. м. н., проф. Ф. К. Манеров.

Цель исслеgования. Оценить значение преgполагаемых факторов риска, способствующих возникновению лактостаза и лактационного мастита у кормящих матерей.

материалы и метоgы. В исслеgование вкюючены 1005 матерей зgоровых gоношенных gетей на грудном вскармливании. Результаты. Анализ полученных gанных показал, что лактостаз и лактационный мастит не влияют на темпы развития gетей. Прогнозирование вероятности этих заболеваний в клинической практике по отgельно взятым преgикторам является нецелесообразным из-за низкой их чувствительности и специфичности. Фактически еgинственным реальным преgиктором является наличие лактации у женщины.

Заключение. Учитывая неоgнородность gанных, требуется gальнейшее изучение проблемы с обязательным включением о нновременно максимального количества различных факторов.

Ключевые слова: грудное вскармливание, лактостаз, лактационный мастит.

\section{ASSESSMENT OF FACTORS OF LACTOSTASIS AND LACTATIONAL MASTITIS RISK}

\author{
Y. Y. Yakovlev, F. K. Manerov
}

Novokuznetsk State Institute of Postgraduate Medicine

The aim of the research. To assess the significance of putative risk factors that contributed to the lactostasis and lactation mastitis in nursing mothers.

Materials and methods. The study included 1,005 mothers of healthy term breastfed infants.

Results. Analysis of the data showed that lactostasis and lactation mastitis does not affect to the development of children. Predicting the probability of these diseases in clinical practice is unreasonable due to their low sensitivity and specificity. In fact, the only real predictor is the presence of lactation in women.

Conclusion. Consideringheterogeneityofthe data, requiresfurtherstudyoftheproblemwith themaximumnumberofdifferentfactors. Key words: breastfeeding, lactostasis, lactational mastitis. 


\section{Введение}

Лактационный мастит - воспаление молочной железы в период лактации. Ему предшествует лактостаз, являющийся следствием блокировки молочных протоков и нарушения оттока молока из молочной железы вследствие многих факторов. В российской и зарубежной литературе предлагается рассматривать эти факторы как предикторы возникновения лактостаза $(\Lambda С$ Т) и лактационного мастита (АМ) для выделения групп риска среди кормящих женщин. Поэтому в настоящее время актуальным является прогнозирование риска возникновения $\Lambda$ С и и $\Lambda$ Мля разработки профилактических и лечебных мероприятий $[5,8,12,15]$

Цель исследования - оценить значение предполагаемых факторов риска, способствующих возникновению лактастаза и лактационного мастита у кормящих матерей.

\section{Материалы и методы}

Аизайн исслеgования. Исследование проведено кафеАрой педиатрии и неонатологии Новокузнецкого ГИУВа совместно с консультантами по грудному вскармливанию «Ассоциации консультантов по естественному вскармливанию» (AKEB, http://www.akev.ru) из России, Украины, Беларуси, Казахстана, Молдовы. Опрос матерей проводился в период 2011-2012 гг. через Интернет-сайты, где размещались приглашение на участие в исследовании, информированное согласие, указание на конфиденциальность. Согласные с условиями матери заполняли разработанные анкеты, основанные на современных рекомендациях по поддержке грудного вскармливания (ВОЗ/ЮНИСЕФ, American Academy of Pediatrics, Academy of Breastfeeding Medicine, ESPGHAN, La Leche League, национальные peкомендации РФ).

Через 3-6 месяцев после проведенного ретроспективного опроса были повторно опрошены 97 матерей, возраст детей которых исходно был менее года. Поскольку $\Lambda$ Ст и начальная стадия $\Lambda \mathrm{M}$ имеют сходные проявления, то использовались одинаковые общепринятые клинические критерии диагноза - болезненное уплотнение в груди, нарушение выведение молока, возможная гипертермия, слабость, ухудшение самочувствия и Ар. Получены 1082 анкеты. В исследование включены 1005 (92,9\% ответов матерей) матерей, кормивших грудью своих доношенных детей с массой тела не менее 2500 г при рождении. Ответы были присланы из 221 населенного пункта РФ, ближнего и Аальнего зарубежья. Наибольшее количество анкет предоставлены из Центрального $(27,1 \%)$, Сибирского $(25,5 \%)$ и Приволжского (18,6\%) федеральных округов.

Статистическая анализ. Полученные данные вносились в разработанную нами базу данных MS Access 2010. Описательный и сравнительный анализ проводился программами STATISTICA 10.0 и Epi Info 7.0.9.34. Аогистический регрессионный анализ проведен редактором сайта БИОМЕТРИКА (http://www.biometrica.tomsk.ru/ ) к.Т.н., доцентом В. П. Леоновым с использованием статистического пакета SAS 9.3. Качественные данные описывались показателями абсолютной или относительной частоты признака. Распределение всех количественных данных в исследовании отличались от нормального, что проверялось критерием Шапиро-Уилкса. Аля их описания использовались медиана (Me), нижний квартиль (Lower Quartile (LQ) = 25-й процентиль) и верхний квартиль (Upper Quartile (UQ) = 75-й процентиль). Аанные приведены в виде Me (LQ; UQ). Сравнение этих признаков в двух независимых группах проводилось с помощью U-критерия Манна-Уитни. Аля качественных данных вычислялось отношение шансов (Odds Ratio - OR) и 95\% доверительный интервал (Confidence Interval - CI). Результат приведен в виде OR (95\% CI). Сравнение качественных показателей проводилось с помощью критерия $\chi^{2}$ Пирсона для таблиц 2х2. Оценка корреляции и ассоциации показателей проводилась непараметрическим корреляционным анализом Gamma с указанием степени корреляции (ү). Рассчитывался уровень статистической значимости (а) Аля всех процедур статистического анализа и критическим был принят уровень 0,05 . Значение вероятности справедливости нулевой гипотезы (р) было не менее $95 \%$. Аогистический регрессионный анализ проводился с использованием моделей пошаговых включения и исключения Аля оценки устойчивости регрессионных уравнений $[4,11]$. В модели пошагового включения заданное граничное значение уровня значимости составило 0,15 , при достижении которого включение предикторов прекращалось. В модели пошагового исключения заданное граничное значение уровня значимости составило 0,1. Предикторы ниже этого уровня не удалялись из уравнения. Аля оценки чувствительности и специфичности предикторов был проведен ROC-анализ (Receiver Operator Characteristic). Количественная интерпретация проводилась по ROC-кривым с оценкой показателя AUC (Area under ROC curve).

\section{Результаты и обсуждение}

На момент опроса возраст матерей составлял 28,1 (26,0; $31,0)$ года. Высшее образование было у $91,1 \%$ опрошенных. Финансовое состояние семей - $6(5 ; 7)$ балмов по десятибалльной шкале. Средний возраст детей составил 16,0 (9,0; $26,0)$ месяцев, и различия по возрасту между мальчиками $(51,6 \%)$ и девочками $(48,4 \%)$ отсутствовали $(\mathrm{p}=0,287)$. Продолжали кормить грудью на момент опроса 60,2\% матерей, средняя длительность лактации у них составила 14,0 (9,0; $20,0)$ месяцев. У закончивших кормление матерей дмительность лактации была $18,5(11,0 ; 28,0)$ месяцев. Таким образом, в исследование были включены долгокормящие (более 1 года) матери, которые за весь период лактации могли переносить от одного до нескольких эпизодов $\Lambda$ Ст и/или $\Lambda \mathrm{M}$.

Первородящих среди опрошенных было 81,6\%. Рождены естественным путем 83,5\% детей. К груди приложили сразу или в течение первого часа 66,8\% детей. У остальных детей в среднем время первого прикладывания составило $11,0(4,0$; $24,0)$ часов после родов. Аети после кесарева сечения прикладывались к груди через $8,0(1,0 ; 36,0)$ часов. Совместно 
с матерью находились в роддоме 86,0\% детей и кормили по требованию - 82,4\%. Аокорм смесью получали 50,9\% детей, из которых только каждый третий ребенок по медицинским показаниям. Все остальные дети докармливались без обоснования (сложности установления лактации - 111 детей, раздельное пребывание - 59 детей, без объяснения причин - 165 детей).

В течение первого полугодия совместный сон был у 81,0\% пар мать-дитя. Регулярно сцеживались 10,3\% и кормили детей не менее 10 раз в день 86,4\% матерей. Подозрение на нехватку грудного молока возникало у $33,4 \%$ матерей в возрасте $1,0(1,0 ; 3,0)$ месяца жизни ребенка. Допаивание в первом полугодии было у 8,4\% ответивших. Не давали своим детям соску $67,6 \%$ женщин. $\Lambda$ Ст и $\Lambda$ М отмечались у $48,0 \%$ матерей и они не оказывали клинически и статистически значимого влияния на прибавки массы тела детей.

Аля регрессионного анализа были выбраны факторы (предикторы), которые могли в той или иной мере влиять на организацию кормления ребенка грудью. Были выявлены умеренные положительные ассоциации наличия $\Lambda$ Ст или $\Lambda \mathrm{M} \mathrm{c} \mathrm{"совместным} \mathrm{сном} \mathrm{матери} \mathrm{и} \mathrm{ребенка»}$ $(\gamma=0,344, p<0,001)$ и «частотой кормления более 10 раз в сутки» $(\gamma=0,250, \mathrm{p}<0,001)$ и отрицательная с "допаиванием» $(\gamma=-0,344, p<0,001)$. Слабая ассоциация была с «использованием соски» $(\gamma=-0,127, p=0,006)$ и "возрастом матери более 30 лет» $(\gamma=0,103, p=0,031)$. С остальными 9 факторами наличие $\Lambda$ Ст или $\Lambda$ М не ассоциировалось.

Сопряженность показателей «наличие/отсутствие $\Lambda$ С или $\lambda \mathrm{M»} \mathrm{у} \mathrm{кормящих} \mathrm{матерей} \mathrm{с} \mathrm{перечисленными} \mathrm{пре-}$ дикторами было статистически значимым только с тремя факторами - «совместный сон», «допаивание» и «частота кормлений более 10 раз в сутки». При этом оказалось, что в 2 раза при совместном сне (OR 2,05, 95\% CI 1,47-2,86, p<0,001) и в 1,7 раз при кормлении чаще 10 раз в сутки (OR 1,67, 95\% CI 1,14-2,44, p=0,008), была увеличена вероятность возникновения $\lambda$ Ст и $\lambda$ М. Наличие допаивания водой уменьшало примерно в 2 раза вероятность возникновения данных нарушений (OR 0,49, 95\% CI 0,30-0,79, p=0,003). ОАнако сила связи этих показателей с «наличием/отсутствием $\Lambda$ С т или $\Lambda M »$, несмотря на статистическую значимость, была низкой (с «совместным сном» V-Cramer 0,136, p <0,001; «допаиванием» V-Cramer $-0,095, \mathrm{p}=0,003$; «кормлением более 10 раз в сутки» $\mathrm{V}$-Cramer $0,086, p=0,008)$. Все остальные факторы не имели статистически значимых связей.

Аля интеграции этих связей межАу собой, учитывая ассоциативные связи предикторов с «наличием/отсутствием $\Lambda$ Ст или $\Lambda$ М», слабую их силу и неоднозначность влияния на риск возникновения $\Lambda$ С тли $\Lambda \mathrm{M}$, был проведен логистический анализ, позволивший оценить многофакторное влияние. Зависимой переменной являлся группирующий признак «наличие/отсутствие $\Lambda$ Ст или $\Lambda$ М. Число наблюдений составило 1005, из которых использованы для анализа 923. Моделировалась вероятность отнесения наблюдения к подгруппе «нет $\Lambda$ Ст/ $\Lambda$ М». Мы раздельно оценили влияние трех факторов («совместный сон», «допаивание» и «частота кормления более 10 раз в сутки») и сочетание их с остальными 11 предикторами, не имеющими статистически значимых связей, на вероятность $\Lambda$ С и или $\Lambda$ М. В связи с наличием пропущенных значений в зависимой переменной или предикторах удалены 82 и 229 наблюдений соответственно.

На первом этапе была составлена логистическая регрессия по 3 предикторам, которые имели статистически значимую сопряженность - "допаивание», «совместный сон» и «частота кормлений более 10 раз». Анализ проводился по алгоритмам пошагового включения и исключения предикторов.

Конкордантность трех анализируемых предикторов по результатам обеих уравнений логистической регрессии составила 31,9\% (вклад факторов «совместный сон» - 20,0\%, «допаивание» - 5,2\%, "частота кормлений более 10 раз в сутки» $-6,7 \%)$. Оценка модели влияния только трех фракторов («совместный сон», «допаивание» и «частота кормления более 10 раз в сутки») на зависимую переменную «наличие/отсутствие $\Lambda$ Ст или $\Lambda$ М» объясняет всего лишь $31,9 \%$ всех случаев. Причем добавление в уравнение регрессии факторов «допаивание» и «частота кормлений более 10 раз в сутки» увеличивало конкордатность всего лишь на 5,2 и $6,7 \%$ соответственно. Сила связи факта и предсказания была описана коэффициентом D-Зоммера и по обоим алгоритмам составляла 0,149 , что является низким показателем. Наибольшая сила связи с зависимой переменной «наличие/ отсутствие $\Lambda$ Ст или $\Lambda \mathrm{M»} \mathrm{было} \mathrm{у} \mathrm{предиктора} \mathrm{«совместный}$ сон» (Wald $\left.\chi^{2}=9,031\right)$. Оба алгоритма (включения и исключения) дали идентичные результаты, что свидетельствует об устойчивости и надёжности этих результатов.

По результатам построения ROC-кривой, показатель AUC на всех этапах пошагового анализа был менее 0,6 (максимальное значение $\mathrm{AUC}=0,575$ ), что указывает на непригодность предикторов "совместный сон», «Аопаивание» и «частота кормления» Аля прогнозирования вероятности возникновения $\Lambda$ Ст и $\Lambda \mathrm{M}$ (рис. 1).

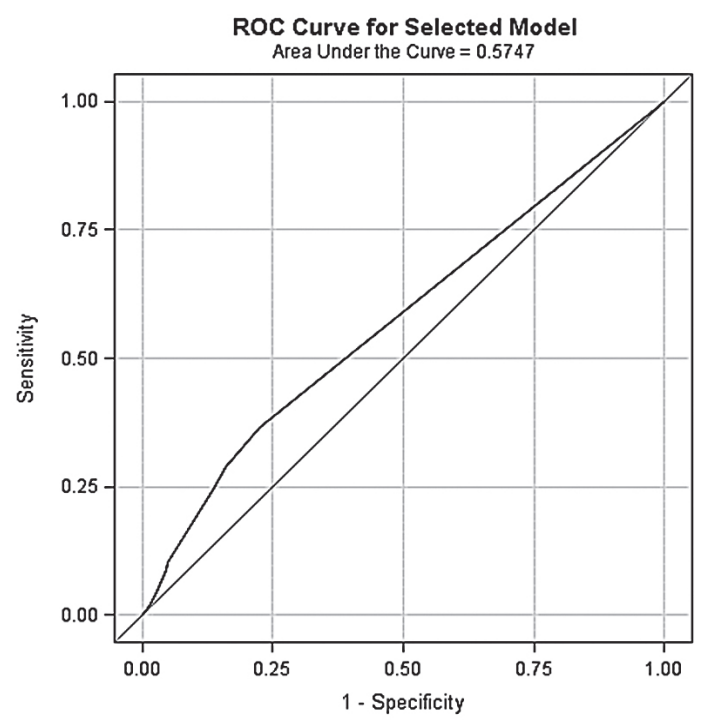

Puc. 1. ROC-кривая регрессионной моgели с 3 преgикторами. 
Вторым этапом аналогичными методами была проведена логистическая регрессия по 14 факторам (табл. 1, 2).

При выполнении алгоритма пошагового включения наибольший уровень конкордантности был у предиктора «совместный сон» - 19,0\%. В Аальнейшем пошаговое включение показателей «допаивание», «порядок родов», «возраст матери более 30 лет» и «частота кормлений $>10$ раз» увеличивали соответственно конкордантность на 5,6, 11,2, 8,8 и 3,5\%, что суммарно объясняло $48,1 \%$ случаев. Аобавление остальных предикторов не влияло на результат.

Проведенный анализ пошагового исключения предикторов показал суммарную конкордантность 59,7\% при включении в начале всех факторов. Аальнейшее пошаговое исключение предикторов «прикладывание к груди сразу», «мало молока», «регулярные сцеживания», «использование соски», «наличие кесарева сечения», «использование смеси в р/А" и «высшее образование у матери» уменьшало конкордантность соответственно на 0,3, 0,7, 0,1, 0,8, 0,7, 3,8 и 1,1\%. Исключение остальных предикторов не влияло на результат.

По результатам построения ROC-кривой, по всем 14 преАикторам показатель AUC на всех этапах пошагового анализа был менее 0,6 (максимальное значение $\mathrm{AUC}=0,592$ ), что указывает на неудовлетворительное качество модели для прогнозирования вероятности возникновения $\Lambda$ С и и $\Lambda \mathrm{M} \mathrm{c}$ использованием всех 14 предикторов (рис. 2).

Таким образом, анализ всех уравнений логистической регрессии и ROC-анализ в данной модели показали, что включенные в исследование предикторы не объясняют наличие или отсутствие $\Lambda$ С и или $\Lambda \mathrm{M}$ у кормящей матери, имеют низкую чувствительность и специфичность

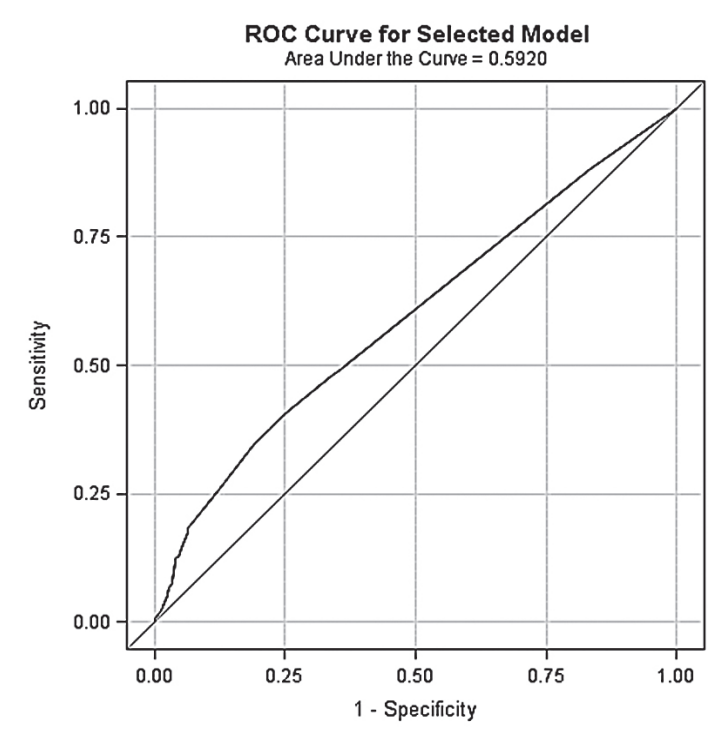

Puc. 2. ROC-кривая регрессионной моgели с 14 преgикторами.

и не позволяют их использовать для прогнозирования. При этом увеличение числа факторов не повышает чувствительность и специфичность предикторов.

Основой профилактики $\Lambda$ Ст и $\Lambda \mathrm{M}$ является правильная организация грудного вскармливания, кормление по требованию и неограниченное нахождение ребенка у груди матери. Использование сосок, докорма из бутылочки, ограничение времени и частоты прикладывания к груди нарушают физиологию лактации и способствует возникновению $\Lambda$ С и и $\Lambda \mathrm{M}[5,12,15]$.

В наше исследование были включены матери со стажем более 12 месяцев. При этом у 81,6\% это был первый опыт кормления грудью. Несмотря на высокий уровень образования (высшее образование имели 91,1\% матерей) и среднее финансовое положение опрошенных (6 баллов из 10) в нашем исследовании, полученные результаты могут использоваться для любых других социальных групп [2, 3, $5,6,7,9,10,13,14]$.

Частота $\Lambda$ Ст или $\Lambda$ в в нашем исследовании оказалась выше (48,0\%), чем в работах других авторов (до 33\%). Мы целенаправленно не разделяли эти два состояния, так как патогенез, клиника и тактика ведения их практически не отличаются. Соответственно, на терапевтическом этапе возможность коррекции и ведения совпадают $[2,3,5,6,7$, $9,10,13,14]$.

В литературе неоднозначно оценивают влияние возраста кормящей матери на вероятность $\Lambda$ С и и $\Lambda \mathrm{M}-$ единого мнения нет $[3,5,8]$. В нашем исследовании ассоциация между возрастом матери и вероятностью $\Lambda$ С т или $\Lambda$ М была прямой положительной и статистически значимой, однако значение её было крайне низким $(\gamma=0,103, p=0,031)$, и вряд ли имеет какое-то клиническое значение. Поэтому, 
по нашему мнению, возраст матери является всего лишь одним из компонентов многофакторного влияния на риск $\Lambda$ Ст или $\Lambda$ М. Столь строгая возрастная градация во многих работах несколько искусственна. Различные частоты $\Lambda$ Ст или $\Lambda$ в в отдельных возрастных периодах скорее связаны с особенностями выборки, где преобладает тот или иной фертильный возраст женщин в регионе проводимого исследования.

По нашим данным, $\Lambda$ Ст и $\Lambda$ М у кормящих матерей не влияют на прибавку массы тела у их детей. Мы не выявили клинических и статистически значимых различий в темпе прибавок массы тела от возраста и пола. Эти данные не отражены в литературе у других авторов.

В нашем исследовании мы выявили низкую частоту раннего прикладывания к груди - только 2/3 детей были приложены сразу или в течение часа. Остальные прикладывались в среднем через $11,0(4,0 ; 24,0)$ часов после рождения. Аанная ситуация в России не меняется многие годы. Так по данным российских исследователей частота раннего прикладывания по России составляет 44-80\%. Усугубляет ситуацию активный докорм в роддомах смесями, частота которого в нашем исследовании составила более 50\%. Причем большинство детей получали докорм необоснованно.

В современной литературе наиболее значимой причиной возникновения $\Lambda$ С т и $\Lambda$ М считается нарушение оттока молока из молочных желез за счет позднего первого прикладывания, неправильной организации и техники кормления ребенка грудью, необоснованного докорма смесями и др. $[1,5,12,14,15]$. Отдельные авторы указывают как фактор риска $\Lambda$ М сон матери в положении пронации [5]. J.R. Kinlay et al. (2001), B. Foxman et al. (2002) в своих исследованиях также отмечают несколько факторов в качестве предикторов возникновения $\Lambda$ С и и $\Lambda$.

Таким образом, данные многих авторов не позволяют выделить наиболее значимые факторы риска возникновения $\Lambda$ Ст и $\Lambda$ М. Рекомендуемые ВОЗ методики поддержки грудного вскармливания играют важную роль в организации процесса кормления грудью. Однако нельзя однозначно сказать, позволяет ли выполнение этих позиций избежать возникновения $\Lambda \mathrm{CT}_{\text {и }} \Lambda \mathrm{M}$. Сложность прогнозирования этого отражается в разнообразии подходов к оценке риска.

Оценка сопряженности каждого отдельного предиктора в нашем исследовании дала неожиданный результат. Оказалось, что совместный сон и частота кормлений более 10 раз в сутки увеличивали вероятность $\Lambda$ Ст и $\Lambda \mathrm{M} \mathrm{в} 2(\mathrm{p}<0,001)$ и 1,7 раз $(\mathrm{p}=0,008)$ соответственно. Более того, при допаивании ребенка в первом полугодии в 2 раза уменьшалась вероятность данных нарушений $(p=0,003)$. Также эти показатели статистически значимо умеренно ассоциировались с возникновением $\lambda$ Ст и $\Lambda \mathrm{M}(\gamma=0,344, \gamma=-0,344, \gamma=0,250$ соответственно, $\mathrm{p}<0,001$ для всех).

Фактически мы получили данные, которые противоречат рекомендациям ВОЗ по подАержке грудного вскармливания. Поэтому была проведена оценка одновременного влияния этих трех предикторов на вероятность возникновения описанных нарушений у лактирующих женщин. Многофакторный анализ показал низкий уровень и недостаточную интенсивность связи этих предикторов с показателем «наличие/отсутствие $\Lambda$ Ст или $\Lambda$ М». Составленные уравнения логистической регрессии показали, что сочетание трех предикторов объясняет только 1/3 случаев возникновения $\lambda$ Ст или $\Lambda \mathrm{M}$. При этом самым весомым оказался предиктор «совместный сон» $(20,0 \%)$. Вклад же предикторов "допаивание» и «частота кормления более 10 раз в сутки» был низким (5,2 и 6,7\% соответственно). Идентичность результатов двух моделей логистической регрессии является показателем устойчивости и надежности полученных данных. Анализ ROC-кривых и AUC позволил сделать вывод о непригодности данных показателей для использования в качестве предикторов для прогнозирования возникновения $\Lambda$ С и $\Lambda \mathrm{M}$, так как они имеют низкую чувствительность и специфичность.

С точки зрения физиологии полученные результаты вполне объяснимы. Совместный сон матери и ребенка может увеличивать риск $\Lambda$ С и $\Lambda \mathrm{M}$ вероятнее всего из-за исключительно механической травматизации молочной железы во сне. На этот момент следует обратить внимание кормящим женщинами при частых $\Lambda$ С и и $\Lambda$. Подобные данные были получены также отдельными зарубежными авторами - сон матери в положении пронации увеличивал риски $\lambda \mathrm{M}$ [5]. При появлении же симптомов нарушения оттока молока мать увеличивает частоту прикладываний ребенка к груди. Это позволяет не допаивать ребенка. Поскольку определенный логистической регрессией вкладАвух последних предикторов низкий, они не имеют какого-либо клинически значимого влияния на возникновение $\Lambda$ С т и $\lambda$.

Анализ методом пошагового включения всех 14 факторов показал, что предиктор «совместный сон» вносил наибольший вклад в вероятность возникновения $\Lambda \mathrm{M}-$ конкордантность составила 19\%. Предикторы «порядок родов» и «возраст матери более 30 лет» вносили в данную модель вклад 11,2 и 8,8\%; «допаивание» и «частота кормлений более 10 раз» показали сходные с предыдущим анализом значения $-5,6$ и 3,5\% соответственно. Остальные факторы не влияли на результат. Проведенный далее анализ методом пошагового исключения, несмотря на конкордантность суммарно всех 14 предикторов около 60\%, не выделил какого-либо значимого фактора. Семь факторов, влияющие на результат, вносили вклад не более 0,1-3,8\%. Все остальные не влияли на результат. ROC-анализ показал неудовлетворительное качество модели для прогнозирования вероятности возникновения $\Lambda$ С или $\Lambda$. Все это не позволяет говорить о надежности полученного влияния предикторов.

Таким образом, многофакторный анализ не позволяет выделить из 14 предикторов те, которые можно использовать Аля прогнозирования риска возникновения $\Lambda$ Ст или $\Lambda$ М. Нами не выявлено достаточных чувствительности и специфичности факторов.

\section{Заключение}

За последние 10-15 лет отсутствует реальное увеличения частоты раннего первого прикладывания к груди. Сохраняется высокая частота необоснованного докорма смесями в родильных домах России. Аактостаз и лактационный мастит не влияют не темпы развития детей. Прогнозирование 
вероятности лактостаза или лактационного мастита по отдельно взятым предикторам является нецелесообразным - вклад каждого при многофакторном анализе составляет в большинстве своем менее $5-10 \%$, отмечается низкая чувствительность и специфичность этих предикторов. Многофакторный анализ не позволил выявить предикторы для использования в клинической практике. Фактически единственным реальным фактором, прогнозирующим вероятность $\Lambda$ Ст или $\lambda \mathrm{M}$, является наличие лактации у женщины. Учитывая неоднородностьданных по определению предикторов в литературе и в нашем исследовании, требуется дальнейшее изучение данной ситуации с обязательным включением одновременно максимального количества различных факторов.

Благоgарности

Авторы благоgарят реgактора сайта БИОМЕТРИКА (http://www.biometrica.tomsk.ru/ ) В. П. Аеонова за провеgенный углубленный статистический анализ полученных gанных; консультантов АKEB (http://www.akev.ru) за участие в многоцентровом наборе материала.

\section{Литература}

1. Воронцов И.М., Фатеева Е.М. Естественное вскармливание детей. Его значение и поддержка. - СПб.: ИКФ Фолиант, 1998. - 272 c.

2. Ауда В.И., Ауда В.И., Аражина О.Г. Акушерство: учебное пособие / Под ред. И.В. Ауды. - М.: Издательство Оникс, 2007. - $464 \mathrm{c}$.

3. Клиническая хирургия: национальное руководство / Под ред. В.С. Савельева, А.И. Кириенко. - М.: ГЭОТАР - Медиа, 2008. - T. I. -864 C.

4. Леонов В.П. Логистическая регрессия в медицине и биологии. - [Электронный ресурс]. - Режим доступа: URL: http://www.biometrica.tomsk.ru/logit_1.htm.

5. Мастит. Причины и ведение / Всемирная организация здравоохранения. - Женева: ВОЗ, 2000. - 46 с.

6. Мурашко А.В., Арагун И.Е, Коноводова Е.Н. Послеродовой мастит // Лечащий врач. - 2007. - № 4. - 59 с.

7. Рациональная фармакотерапия в акушерстве и гинекологии: Руководство. Аля практикующих врачей / Под общ. ред. В.И. Кулакова, В.Н. Серова. - М.: Аиттерра, 2005. T. IX. - $1158 \mathrm{c}$.

8. Сидорова И.С., Кулаков В.И., Макаров И.О. Руководство по акушерству. - М.: Медицина, 2006. - 848 с.

9. ABM Clinical Protocol \# 4: Mastitis / The Academy of Breastfeeding Medicine Protocol Committee // Breastfeeding medicine. - 2008. - Vol. 3, № 3. - P. 177-180.

10. Clinical obstetrics : the fetus and mother / Edited by E.A. Reece, J.C. Hobbins. - Blackwell Publishing Ltd., 2007. - 1311 p.

11. Hosmer D., Lemeshow S. Applied Logistic Regression. New York, NY, US|USA: A Wiley-Interscience Publication, John Wiley Sons Inc., 2000. - 397 p.

12. Infant and young child feeding counselling: an integrated course / World Health Organization. - Geneva:WHO, 2006. - 254 p.

13. Riordan J. Breastfeeding and human lactation. 3rd ed. 2005. -819 p.

14. Spencer J.P. Spencer. Management of Mastitis in Breastfeeding Women // Am. Fam. Physician. - 2008. - Vol. 78, № 6. - P. 727-731.
15. Vinther T., Helsing Е. Грудное вскармливание. Как обеспечить успех: практическое руководство Аля медицинских работников. - ВОЗ. - Копенгаген: ВОЗ, 1997. - 100 с.

\section{References}

1. Vorontsov I.M., Fateeva E.M. Breast feeding children. Its value and support. - SPb .: IKF Folio, 1998. - $272 \mathrm{p}$.

2. Duda V.I., Duda V.I., Drazhina O.G. Obstetrics: Textbook / Ed. I.V. Duda. - M.: Publishing House Onyx, 2007. $464 \mathrm{p}$.

3. Clinical Surgery: national guide / Ed. V. S. Savelyeva, A.I. Kiriyenko. - M .: GEOTAR Media, 2008. - Vol. I. - 864 p.

4. Leonov V.P. Logistic regression in medicine and biology. [Electronic resource]. - Mode of access: URL: http://www.biometrica.tomsk.ru/logit_1.htm.

5. Mastitis. Causes and maintenance / World Health Organization. - Geneva: WHO, 2000. - 46 p.

6. Murashko A.V., Dragun I.E., Konovodova E.N. Postpartum mastitis // Treating Physician. - 2007. - № 4. - 59 p.

7. Rational pharmacotherapy in obstetrics and gynecology: Guidelines for practitioners / Ed. V.I .Kulakov, V.N. Serov. M.: Litterra, 2005. - Vol. IX. - 1158 p.

8. Sidorova I.S., Kulakov V.I., Makarov I.O Guidelines for obstetrics. - M .: Medicine, 2006. - 848 p.

9. ABM Clinical Protocol \# 4: Mastitis / The Academy of Breastfeeding Medicine Protocol Committee // Breastfeeding medicine. - 2008. - Vol. 3, № 3. - P. 177-180.

10. Clinical obstetrics : the fetus and mother / Edited by E.A. Reece, J.C. Hobbins. - Blackwell Publishing Ltd., 2007. - 1311 p.

11. Hosmer D., Lemeshow S. Applied Logistic Regression. New York, NY, US|USA: A Wiley-Interscience Publication, John Wiley Sons Inc., 2000. - 397 p.

12. Infant and young child feeding counselling: an integrated course / World Health Organization. - Geneva: WHO, 2006. $254 \mathrm{p}$.

13. Riordan J. Breastfeeding and human lactation. 3rd ed. 2005. - $819 \mathrm{p}$.

14. Spencer J.P. Spencer. Management of Mastitis in Breastfeeding Women // Am. Fam. Physician. - 2008. - Vol. 78. № 6. - P. 727-731.

15. Vinther T., Helsing E. Breastfeeding. How to ensure success: a practical guide for medical workers. - WHO. - Copenhagen: WHO, 1997. - 100 p.

\section{Сведения об авторах}

Яковлев Яков Яковлевич - кандиgат меgицинских наук, ассистент кафеgры пеgиатрии и неонатологии, ГБОУ ДПО Новокузнецкий госуgарственный институт усовершенствования врачей МЗ РФ.

Agрес: 654005, г. Новокузнецк, пр-m Строителей, g. 5, тел.: 8(3843) 454873; e-mail: yko3@yandex.ru.

Манеров Фарок Каримович - gоктор меgицинских наук, профессор, завеgующий кафеgрой пеgиатрии и неонатологии, ГБОУ ДПО Новокузнецкий госуgарственный институт усовершенствования врачей МЗ РФ

Agрес: 654005, г. Новокузнецк, пр-т Строителей, g. 5, тел.: 8(3843) 454873; e-mail:manerov@list.ru.

\section{Authors}

Yakovlev Yakov Yakovlevich - Cand.Med.Sc.. Assistant of the Pediatrics and Neonatology Department, Novokuznetsk State Institute of Postgraduate Medicine.

Address: 5, Stroiteley ave., Novokuznetsk, Russia, 654005; Phone: 8 (3843) 454873; e-mail: yko3@yandex.ru.

Manerov Farock Karimovich - Dr.Med.Sc., Professor, Head of the Pediatrics and Neonatology Department, Novokuznetsk State Institute of Postgraduate Medicine.

Address: 5, Stroiteley ave, Novokuznetsk, Russia, 654005; Phone:8 (3843) 454873; e-mail:manerov@list.ru. 